\title{
Accurate Solutions to Water Wave Scattering by Vertical Thin Porous Barriers
}

\author{
Ai-jun Li, Yong Liu, and Hua-jun Li \\ Shandong Provincial Key Laboratory of Ocean Engineering, Ocean University of China, Qingdao 266100, China \\ Correspondence should be addressed to Yong Liu; liuyong@ouc.edu.cn
}

Received 1 April 2015; Revised 11 September 2015; Accepted 20 September 2015

Academic Editor: Erik Cuevas

Copyright (C) 2015 Ai-jun Li et al. This is an open access article distributed under the Creative Commons Attribution License, which permits unrestricted use, distribution, and reproduction in any medium, provided the original work is properly cited.

The water wave scattering by vertical thin porous barriers is accurately solved in this study. Two typical structures of a surfacepiercing barrier and a submerged bottom-standing barrier are considered. The solution procedure is based on the multi-term Galerkin method, in which the pressure jump across a porous barrier is expanded in a set of basis functions involving the Chebychev polynomials. Then, the square-root singularity of fluid velocity at the edge of the porous barrier is correctly modeled. The present solutions have the merits of very rapid convergence. Accurate results for both the reflection and the transmission coefficients and wave forces are presented. This study not only gives a promising procedure to tackle wave interaction with vertical thin porous barriers but also provides a reliable benchmark for complicated numerical solutions.

\section{Introduction}

Vertical thin plates have been used as simple breakwaters in coastal engineering due to their merits of simple structure, constructing convenience, and low engineering cost. In some cases, a vertical barrier (plate) can play an important role in protecting coast from erosion as well as various activities along the coastline from wave attack. The practical vertical barriers are often surface-piercing and supported on piles or submerged below the ocean surface and bottom-standing [1-5].

An abundance of analytical studies for linear water wave scattering by vertical thin solid barriers have been conducted. Ursell [6] carried out an early study on normally incident wave transmission by a vertical thin barrier in deep water. Evans [7] developed expressions for the first- and secondorder forces and moments on a submerged vertical thin wall based on linear potential theory. Morris [8] developed a variational approach to normally incident water interaction with two nonsymmetric vertical thin barriers. Losada et al. [9] and Abul-Azm [10] used matched eigenfunction expansion method to develop analytical solutions for obliquely and normally incident wave scattering by vertical thin barriers with different configurations, respectively. Kriebel and Bollmann [11] compared the transmission coefficients of surfacepiercing solid barriers calculated by the wave power theory (an approximate method) and the matched eigenfunction expansion method. Porter and Evans [1] investigated oblique wave scattering by various partial solid barriers using a multiterm Galerkin method and obtained the upper and lower boundaries of reflection and transmission coefficients with extremely high accuracy. Banerjea et al. [12] also used the multi-term Galerkin method to obtain accurate solutions for oblique wave scattering by single and double submerged vertical solid barriers with gaps. Moreover, the multi-term Galerkin method has been used by Evans and Porter [13], Martins-Rivas and Mei [14], and Chang et al. [15] for threedimensional vertical thin walls. Compared with matched eigenfunction expansion method, the beauty of multi-term Galerkin method is that it can correctly model the squareroot singularity of fluid velocity near the edge of vertical plate. Then, extremely accurate results of hydrodynamic quantities can be obtained.

Besides good shelter function with required transmission coefficient, smaller wave force acting on a barrier is expected in engineering design for ensuring the safety of the structure. Thus, porous barriers have been proposed and used as 


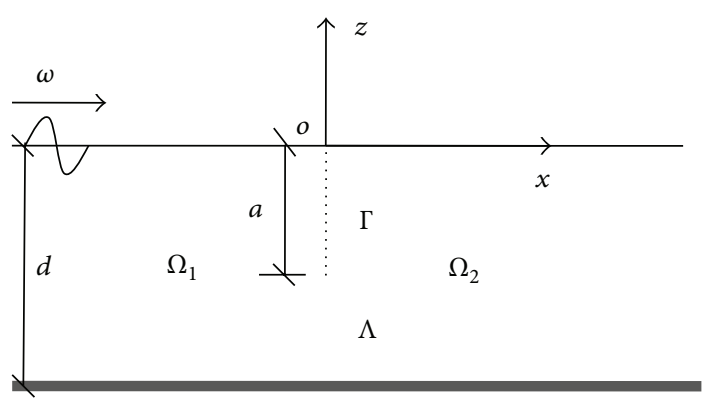

(a) A surface-piercing porous barrier (Type 1)

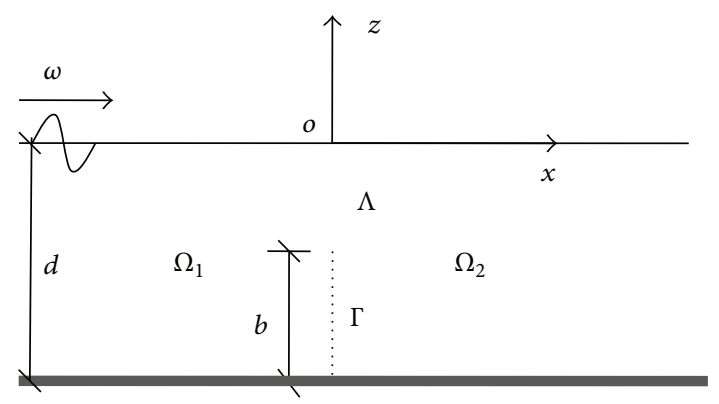

(b) A submerged bottom-standing porous barrier (Type 2)

FIGURE 1: Idealized sketch of water wave scattering by vertical porous barriers.

breakwaters in coastal engineering. After generalizing the study of Tuck [16] for permeable thin barriers in deep water, Macaskill [17] examined normally incident wave interaction with a permeable thin barrier in finite water depth by solving integral equations. Sahoo [18] applied a perturbation analysis to study water wave scattering by porous barriers with small porosity effect in deep water. Isaacson et al. [2] used matched eigenfunction expansion method to develop an analytical solution for normally incident wave interaction with a partially immersed porous barrier and validated their solution using experimental data. Lee and Chwang [19] examined wave scattering and radiation by vertical thin porous barriers with four different configurations based on the eigenfunction expansion and least square methods. They found that due to the energy dissipation by holes, the reflection coefficient and wave force of a porous barrier are smaller than those of a solid barrier. Sahoo et al. [20] generalized the solution of Lee and Chwang [19] to obliquely incident waves. Recently, Karmakar and Guedes Soares [21] developed analytical solution for wave transformation due to multiple bottom-standing flexible porous barriers using eigenfunction expansion method. Gayen and Mondal [22] studied normally incident wave reflection and transmission by a submerged inclined porous barrier by solving a second kind hypersingular integral equation.

As mentioned above, a variety of solutions with respect to vertical thin porous barriers have been developed. But rapidly convergent solutions for thin porous barriers are still lacking. In particular, for multiple barriers, a rapidly convergent solution is of great significance for parameter studies. Also, an accurate analytical solution can serve as a reliable benchmark for complicated numerical potential flow solutions. Thus, we will develop extremely accurate solutions for vertical thin porous barriers in this study. As a first step, we will consider water wave scattering by two simple and typical thin porous barriers, that is, a surface-piercing barrier and a submerged bottom-standing barrier. We use the multiterm Galerkin method [1] to incorporate the square-root singularity of fluid velocity near the edge of porous barrier into the solution. The present solution procedure can be easily extended to more complicated structures, such as various multiple porous barriers.
The governing equation and boundary conditions of the present problem are given in the following section. In Section 3, the solving procedure using multi-term Galerkin method is detailed. In Section 4, the rapid convergence and high accuracy of the present solution are shown by comparing with matched eigenfunction expansion solution. Then, we present some typical curves of hydrodynamic quantities to show the effects of major barrier parameters. Finally, the main conclusions of this study are drawn.

\section{The Boundary Value Problem}

The idealized sketch of water wave scattering by vertical porous barriers is given in Figure 1. The vertical porous barrier is surface-piercing (Type 1) or totally submerged and bottom-standing (Type 2). The thickness of the porous barrier is assumed to be zero, as it is very small compared with the incident wavelength. The water depth is $d$. We use $\Gamma$ to denote the area of barrier and $\Lambda$ to denote the fluid area of gap. A Cartesian coordinate system with the $z$-axis taking vertically upwards along the barrier and the $x$-axis along the still water level is used for mathematical descriptions. The incident waves propagate along the positive $x$-direction.

It is assumed that the fluid is inviscid and incompressible, the fluid motion is irrotational, and the incident harmonic wave is of small amplitude with angular frequency $\omega$. Then, a velocity potential $\Phi(x, z, t)$ can be used to describe the fluid motion. We separate out the time factor $\mathrm{e}^{-\mathrm{i} \omega t}$ and then write the velocity potential and the dynamic pressure as

$$
\begin{aligned}
& \Phi(x, z, t)=\operatorname{Re}\left[-\frac{\mathrm{i} g A}{\omega} \phi(x, z) \mathrm{e}^{-\mathrm{i} \omega t}\right], \\
& P(x, z, t)=\operatorname{Re}\left[-\frac{\mathrm{i} g A}{\omega} p(x, z) \mathrm{e}^{-\mathrm{i} \omega t}\right],
\end{aligned}
$$

where Re denote the real part of function; $i=\sqrt{-1} ; g$ is the gravitational acceleration; $A$ is the incident wave amplitude; $t$ is the time; $\phi(x, z)$ and $p(x, z)$ are, respectively, the spatial velocity potential and dynamic pressure.

For the convenience of study, the whole fluid domain is divided into two sub-regions: left sub-region $\Omega_{1}(-d \leq z \leq$ $0,-\infty<x<0)$ and right sub-region $\Omega_{2}(-d \leq z \leq 0,0<$ 
$x<+\infty)$. The spatial velocity potential in each sub-region satisfies the Laplace equation:

$$
\frac{\partial^{2} \phi_{j}(x, z)}{\partial x^{2}}+\frac{\partial^{2} \phi_{j}(x, z)}{\partial z^{2}}=0, \quad j=1,2,
$$

where the subscript $j$ denotes variables in sub-region $j$. The velocity potentials also satisfy the following boundary conditions on the free surface, the water bottom, and the far fields:

$$
\begin{aligned}
\frac{\partial \phi_{j}}{\partial z} & =\frac{\omega^{2}}{g} \phi_{j} \\
z=0, j=1,2, & \\
\frac{\partial \phi_{j}}{\partial z} & =0, \quad z=-d, j=1,2, \\
\lim _{x \rightarrow+\infty}\left(\frac{\partial \phi_{2}}{\partial x}-\mathrm{i} k_{0} \phi_{2}\right) & =0, \\
\lim _{x \rightarrow-\infty}\left(\frac{\partial}{\partial x}+\mathrm{i} k_{0}\right)\left(\phi_{1}-\phi_{0}\right) & =0
\end{aligned}
$$

where $k_{0}$ is the incident wave number and $\phi_{0}$ is the velocity potential of incident waves.

At the edge of the porous barrier, the gradient of the velocity potential has a square-root singularity ([23, Eq. (2.85)] and [24, Eq. (1e)]):

$$
|\nabla \phi|=o\left(r^{-1 / 2}\right) \quad \text { as } r \longrightarrow 0,
$$

where $r=\sqrt{x^{2}+(z+a)^{2}}$ for Type 1 barrier and $r=$ $\sqrt{x^{2}+(z+d-b)^{2}}$ for Type 2 barrier. In order to develop an accurate solution for the present problem, the square-root singularity of fluid velocity must be correctly modeled. This will be detailed in the following section.

Besides preceding boundary conditions, the velocity potentials satisfy the transmission boundary conditions at the interface of two sub-regions:

$$
\begin{aligned}
\frac{\partial \phi_{1}}{\partial x} & =\frac{\partial \phi_{2}}{\partial x}, \quad x=0, \quad z \in \Lambda, \\
\phi_{1} & =\phi_{2}, \quad x=0, \quad z \in \Lambda, \\
\frac{\partial \phi_{1}}{\partial x} & =\frac{\partial \phi_{2}}{\partial x}=\mathrm{i} k_{0} G\left(\phi_{1}-\phi_{2}\right), \quad x=0, \quad z \in \Gamma,
\end{aligned}
$$

where $G$ is a complex effect parameter of the porous barrier [25]. Equations (8) and (9) denote, respectively, that the horizontal fluid velocities and the dynamic pressures are continuous at the gap. Equation (10) is the boundary condition on the porous barrier, which was developed by Yu [25] based on the classical porous medium model of Sollitt and Cross [26]. The first equals sign in (10) denotes that the normal fluid velocity is continuous at the porous barrier. The second equals sign in (10) denotes that the normal fluid velocity through the porous barrier is proportional to the pressure jump across the barrier. Then, wave energy dissipation is introduced by (10).
According to $\mathrm{Yu}$ [25], the complex porous effect parameter $G$ can be estimated by $G=\varepsilon /\left[k_{0} \delta(f-i s)\right]$, where $\varepsilon, f, s$, and $\delta$ are the porosity, the linearized resistance coefficient, the inertial coefficient, and the thickness of the porous plate (porous medium), respectively. The value of the inertial coefficient $s$ may be simply treated as unity [27, 28]. The value of the linearized resistance coefficient $f$ must be determined by experimental tests. Suh et al. [28] proposed an empirical formula for the resistance coefficient: $f=$ $0.0584(\varepsilon \delta / d)^{-0.7}$. The real and imaginary parts of $G$ denote the linearized resistance effect and the inertial effect of a porous plate, respectively. The resistance effect brings wave energy dissipation, and the inertial effect produces the phase shift of wave motion. When $|G|$ equals zero, the barrier is impermeable. If $|G|$ tends to infinity, the barrier becomes entirely transparent (no porous barrier). This can be observed by rewritten (10) as: $\phi_{1}-\phi_{2}=\widetilde{G} \partial \phi_{1} / \partial x=\widetilde{G} \partial \phi_{1} / \partial x$, where $\widetilde{G}=1 /\left(\mathrm{i} k_{0} G\right)$. In our computations, a very large value of $G=10^{10}$ is adopted for entirely transparent wall.

\section{Methods of Solution}

3.1. Expressions of Velocity Potentials. By the separation of variables, the velocity potentials satisfying the governing equation in (2) and the relevant boundary conditions in (3)(6) can be written as

$$
\begin{aligned}
& \phi_{1}=\left(\mathrm{e}^{\mathrm{i} k_{0} x}+R \mathrm{e}^{-\mathrm{i} k_{0} x}\right) Z_{0}(z)+\sum_{m=1}^{\infty} A_{m} \mathrm{e}^{k_{m} x} Z_{m}(z), \\
& \phi_{2}=T \mathrm{e}^{\mathrm{i} k_{0} x} Z_{0}(z)+\sum_{m=1}^{\infty} B_{m} \mathrm{e}^{-k_{m} x} Z_{m}(z),
\end{aligned}
$$

where $R, A_{m}$, T, and $B_{m}$ are unknown expansion complex coefficients. The wave numbers $k_{0}$ and $k_{m}$ are the positive real roots of the following dispersion relation:

$$
\omega^{2}=g k_{0} \tanh k_{0} d=-g k_{m} \tan k_{m} d, \quad m=1,2, \ldots
$$

In (11), the vertical eigenfunctions $Z_{m}(z)$ are given by

$$
Z_{m}(z)= \begin{cases}\frac{\cosh k_{0}(z+d)}{\cosh k_{0} d}, & m=0, \\ \frac{\cos k_{m}(z+d)}{\cos k_{m} d}, & m=1,2, \ldots\end{cases}
$$

It is noted that the eigenfunctions $Z_{m}(z)$ are orthogonal over $[-d, 0]$ :

$$
\int_{-d}^{0} Z_{m}(z) Z_{n}(z) \mathrm{d} z=0, \quad m \neq n
$$


and integrals of their own square over $[-d, 0]$ are

$$
N_{m}= \begin{cases}\int_{-d}^{0} Z_{0}^{2}(z) \mathrm{d} z=\frac{1}{\cosh ^{2} k_{0} d}\left(\frac{d}{2}+\frac{\sinh 2 k_{0} d}{4 k_{0}}\right), & m=0, \\ \int_{-d}^{0} Z_{m}^{2}(z) \mathrm{d} z=\frac{1}{\cos ^{2} k_{m} d}\left(\frac{d}{2}+\frac{\sin 2 k_{m} d}{4 k_{m}}\right), & m=1,2, \ldots\end{cases}
$$

Then, applying the transmission boundary conditions in (8)-(10), the unknown expansion coefficients in (11) can be determined. For convenience, these transmission conditions are rewritten as

$$
\begin{aligned}
\frac{\partial \phi_{1}}{\partial x} & =\frac{\partial \phi_{2}}{\partial x}, \quad x=0,-d \leq z \leq 0, \\
\frac{\partial \phi_{2}}{\partial x} & =\mathrm{i} k_{0} G\left(\phi_{1}-\phi_{2}\right), \quad x=0, \quad z \in \Gamma \\
\phi_{1} & =\phi_{2}, \quad x=0, \quad z \in \Lambda .
\end{aligned}
$$

Substituting the expressions for the velocity potentials in (11) into (16), we have

$$
\begin{aligned}
& \mathrm{i} k_{0}(1-R) Z_{0}(z)+\sum_{m=1}^{\infty} k_{m} A_{m} Z_{m}(z) \\
& \quad=\mathrm{i} k_{0} T Z_{0}(z)-\sum_{m=1}^{\infty} k_{m} B_{m} Z_{m}(z), \quad-d \leq z \leq 0 .
\end{aligned}
$$

Multiplying both sides of (19) by $Z_{n}(z)$ and integrating with respect to $z$ over $[-d, 0]$ and then using (14), we obtain

$$
\begin{aligned}
R & =1-T, \\
A_{m} & =-B_{m}, \quad m=1,2, \ldots
\end{aligned}
$$

We note that the two conditions in (17) and (18) are known as dual series relations [29]. They can be combined into a mixed boundary condition along the whole water depth (see (A.1)), which can be solved by a least square method [30-32]. Here we use a similar procedure as that for (16) to transform (17) and (18) into a system of linear equations, and then determine all the unknown coefficients. This is just the traditional matched eigenfunction expansion method and is introduced in the Appendix. However, the square-root singularity of fluid velocity is not incorporated into such a solution procedure. As a result, the convergence of the solution is slow. In order to obtain extremely accurate analytical solution, we use the multiterm Galerkin method [1], instead of traditional matched eigenfunction expansion method, to solve the present problem.
3.2. Multi-Term Galerkin Methods. Following Porter and Evans [1, Eqs. (2.52) and (2.63)], we expand the jump of velocity potentials, that is, the pressure jump, at the porous barrier as

$$
\left.\left(\phi_{1}-\phi_{2}\right)\right|_{x=0}= \begin{cases}0, & z \in \Lambda, \\ \sum_{p=0}^{\infty} a_{p} p_{p}(z), & z \in \Gamma,\end{cases}
$$

where $a_{p}(p=0,1, \ldots)$ are unknown expansion coefficients, and $p_{p}(z)$ is given by

$$
\widehat{p}_{p}(z)=p_{p}(z)-\frac{\omega^{2}}{g} \int_{-a}^{z} p_{p}(t) \mathrm{d} t,
$$

with

$$
\widehat{p}_{p}(z)=\frac{2(-1)^{p} \sqrt{a^{2}-z^{2}}}{\pi(2 p+1) a d} U_{2 p}\left(-\frac{z}{a}\right), \quad-a \leq z \leq 0,
$$

for Type 1 barrier, and

$$
\begin{aligned}
& p_{p}(z)=\frac{2(-1)^{p} \sqrt{b^{2}-(d+z)^{2}}}{\pi(2 p+1) b d} U_{2 p}\left(\frac{d+z}{b}\right), \\
&-d \leq z \leq b-d,
\end{aligned}
$$

for Type 2 barrier. In (23) and (24), $U_{n}(x)=\sin ((n+$ 1) $\arccos x) / \sin (\arccos x)$ is the $n$th order Chebychev polynomial of the second kind. According to the expressions of $p_{p}(z),(22)$ satisfies the free surface condition in (3) and the square-root singularity at the lower edge of Type 1 barrier. Equation (24) satisfies the water bottom condition in (4) and the square-root singularity at the upper edge of Type 2 barrier.

Substituting the expressions for the velocity potentials in (11) into (21) and multiplying both sides of the obtained new equation by $Z_{n}(z)$ and then integrating with respect to $z$ over $[-d, 0]$ and using (14) and (15), we have

$$
\begin{aligned}
R & =\left(2 N_{0}\right)^{-1} \sum_{p=0}^{\infty} a_{p} F_{p 0}, \\
A_{m} & =\left(2 N_{m}\right)^{-1} \sum_{p=0}^{\infty} a_{p} F_{p m}, \quad m=1,2, \ldots
\end{aligned}
$$

where 


$$
F_{p m}= \begin{cases}\int_{-a}^{0} p_{p}(z) Z_{0}(z) \mathrm{d} z=\frac{(-1)^{p} I_{2 p+1}\left(k_{0} a\right)}{k_{0} d}, & m=0, \\ \int_{-a}^{0} p_{p}(z) Z_{m}(z) \mathrm{d} z=\int_{-a}^{0} \widehat{p}_{p}(z) \cos k_{m} z \mathrm{~d} z=\frac{J_{2 p+1}\left(k_{m} a\right)}{k_{m} d}, & m=1,2, \ldots\end{cases}
$$

for Type 1 barrier, and

$$
\begin{aligned}
& F_{p m} \\
& = \begin{cases}\int_{-d}^{b-d} p_{p}(z) Z_{0}(z) \mathrm{d} z=\frac{(-1)^{p} I_{2 p+1}\left(k_{0} b\right)}{k_{0} d \cosh k_{0} d}, & m=0, \\
\int_{-d}^{b-d} p_{p}(z) Z_{m}(z) \mathrm{d} z=\frac{J_{2 p+1}\left(k_{m} b\right)}{k_{m} d \cos k_{m} d}, & m=1,2, \ldots,\end{cases}
\end{aligned}
$$

for Type 2 barrier. In (26) and (27), $J_{n}$ and $I_{n}$ denote the Bessel function and the modified Bessel function of order $n$, respectively. Substituting (11) with (20) and (25) into (17), multiplying both sides of the obtained new equation by $p_{n}(z)$, and then integrating with respect to $z$ over $\Gamma$, we obtain

$$
\begin{aligned}
& \sum_{p=0}^{\infty} a_{p}\left[\frac{1+2 G}{N_{0}} F_{p 0} F_{n 0}\right. \\
& \left.\quad+\sum_{m=1}^{\infty} N_{m}^{-1}\left(2 G-\frac{k_{m}}{\mathrm{i} k_{0}}\right) F_{p m} F_{n m}\right]=2 F_{n 0} .
\end{aligned}
$$

We solve (28) by truncating $n$ and $p$ after $N$ terms and obtain the values of $a_{p}(p=0,1, \ldots, N)$. Then, all the expansion coefficients $R, A_{m}, T$, and $B_{m}$ in velocity potentials are determined using (20) and (25).

3.3. Hydrodynamic Quantities. The reflection and transmission coefficients of the vertical porous barrier are calculated, respectively, by

$$
\begin{aligned}
& K_{R}=|R|=\left|\left(2 N_{0}\right)^{-1} \sum_{p=0}^{N} a_{p} F_{p 0}\right|, \\
& K_{T}=|1-R| .
\end{aligned}
$$

The energy loss coefficient is defined as

$$
K_{L}=1-\left(K_{R}^{2}+K_{T}^{2}\right)
$$

The energy dissipation is introduced by the porous boundary condition in (10), which brings pressure loss on the porous barrier. If the barrier is impermeable $(G=0)$, the energy loss coefficient is always zero.

The dynamic pressure jump on the porous barrier is calculated by the linear Bernoulli equation $p(x, z)=\rho g A\left(\phi_{1}-\right.$ $\phi_{2}$ ), where $\rho$ is the water density. Integrating the dynamic pressure jump along the porous barrier, we get the magnitude of horizontal wave force acting on the barrier:

$$
\begin{aligned}
F & =\rho g A \int_{\Gamma}\left[\phi_{1}\left(0^{-}, z\right)-\phi_{2}\left(0^{+}, z\right)\right] \mathrm{d} z \\
& =2 \rho g A\left[R \int_{\Gamma} Z_{0}(z) \mathrm{d} z+\sum_{m=1}^{N} A_{m} \int_{\Gamma} Z_{m}(z) \mathrm{d} z\right]
\end{aligned}
$$

where

$$
\begin{aligned}
& \int_{\Gamma} Z_{m}(z) \mathrm{d} z \\
& = \begin{cases}\frac{\left[\sinh k_{0} d-\sinh k_{0}(d-a)\right]}{k_{0} \cosh k_{0} d}, & m=0, \\
\frac{\left[\sin k_{m} d-\sin k_{m}(d-a)\right]}{k_{m} \cos k_{m} d}, & m=1,2, \ldots, N,\end{cases}
\end{aligned}
$$

for Type 1 barrier, and

$$
\int_{\Gamma} Z_{m}(z) \mathrm{d} z= \begin{cases}\frac{\sinh k_{0} b}{k_{0} \cosh k_{0} d}, & m=0, \\ \frac{\sin k_{m} b}{k_{m} \cosh k_{m} d}, & m=1,2, \ldots, N\end{cases}
$$

for Type 2 barrier. The dimensionless wave force on the barrier is defined as

$$
\begin{aligned}
K_{F} & =\frac{|F|}{F_{0}}, \\
F_{0} & =\frac{2 \rho g A}{k_{0}} \tanh k_{0} d,
\end{aligned}
$$

where $F_{0}$ is the wave force acting on per-unit width in an impermeable vertical wall extending from seabed to the still water level.

\section{Results}

The second part in the left hand side of (28) is still an infinite series after truncating $n$ and $p$. Thus, the values of $F_{p m}$ and $F_{n m}$ need to be estimated by truncating $m$. Porter and Evans [1] have detailed the computation method of this type of series for solid barriers. In this study, all the series are first computed taking 800 terms, and then they are improved by applying the asymptotic values of Bessel functions as those used in Porter and Evans [1]. In order to obtain the required accuracy, we use $10^{8}$ terms in computations of the improvements. 
TABLE 1: Results of $K_{R}, K_{T}$, and $K_{L}$ for Type 1 barrier at $k_{0} a=0.5$ and $G=0$

\begin{tabular}{lccccc}
\hline \multirow{2}{*}{$a / d$} & \multicolumn{3}{c}{ The present results } & \multicolumn{3}{c}{ Porter and Evans (1995) [1] } \\
& $K_{R}$ & $K_{T}$ & $K_{L}$ & $K_{R}$ & $K_{T}$ \\
\hline 0.1 & 0.439284 & 0.898348 & 0 & 0.43928 & 0.89835 \\
0.4 & 0.349212 & 0.937044 & 0 & - & - \\
0.5 & 0.353987 & 0.935250 & 0 & 0.35399 & 0.93525 \\
0.8 & 0.493434 & 0.869783 & 0 & - & - \\
0.9 & 0.602548 & 0.798083 & 0 & 0.60255 & 0.79808 \\
\hline
\end{tabular}

4.1. Special Cases of Solid Barriers. We first consider the special cases of solid barriers when the porous effect parameter $G$ is zero. The calculated results of $K_{R}, K_{T}$, and $K_{L}$ for two types of barriers are given in Tables 1 and 2. Here, the truncated number of $N$ is 7 . It can be seen from Tables 1 and 2 that the wave energy conservation $\left(K_{L}=0\right)$ is well satisfied for solid barriers. For comparisons, the results obtained using Tables 1 and 2 and (2.25) and (2.26) in Porter and Evans [1] are also added in these tables. For solid barriers, the fluid velocity at the gap $\Lambda$ can also be expanded using a similar method to that in (21). The two approaches based on expanding the pressure jump at $\Gamma$ and the fluid velocity at $\Lambda$ for solid barrier can give the lower and upper bounds of hydrodynamic quantities, respectively. Thus, the results of Porter and Evans [1] for solid barriers can be treated as the exact solution. It can be seen from Tables 1 and 2 that the present results are the same as that of Porter and Evans [1] for five-figure accuracy.

4.2. Convergence Examination for Porous Barriers. The main purpose of the results presented below is to demonstrate the high accuracy and rapid convergence of the present multiterm Galerkin solution for porous barriers. The computed results of the reflection coefficient, the transmission coefficient, and the wave force, $K_{R}, K_{T}$, and $K_{F}$, for the surfacepiercing porous barrier (Type 1) and the submerged porous barrier (Type 2) at different truncated number $N$ are listed in Tables 3 and 4, respectively. It can be seen from these tables that for both barriers with different sizes, the convergence of the solution is very rapid. Results with six-figure accuracy can be generally obtained using the truncating number of $N=6$. Results with higher accuracy can also be obtained using larger truncated numbers. Besides results shown in Tables 3 and 4 , our extensive numerical experiments indicate that other values of wave numbers and barrier sizes produce equally accurate results for $K_{R}, K_{T}$, and $K_{F}$. The high accuracy of the present solution is due to the fact that the nature of the square-root singularity near the edge of the porous barrier is correctly modeled. We note that results with six-figure accuracy are presented to show the rapid convergence of the solution. One may adopt less Galerkin terms to obtain results with two- or three-figure accuracy.

For comparisons, the corresponding results calculated by the matched eigenfunction expansion solution (see the Appendix) for porous barriers are listed in Tables 5 and 6 . It can be seen from these tables that, after using a large truncated number of $M=600$, the matched eigenfunction expansion solution can only give results with three-figure

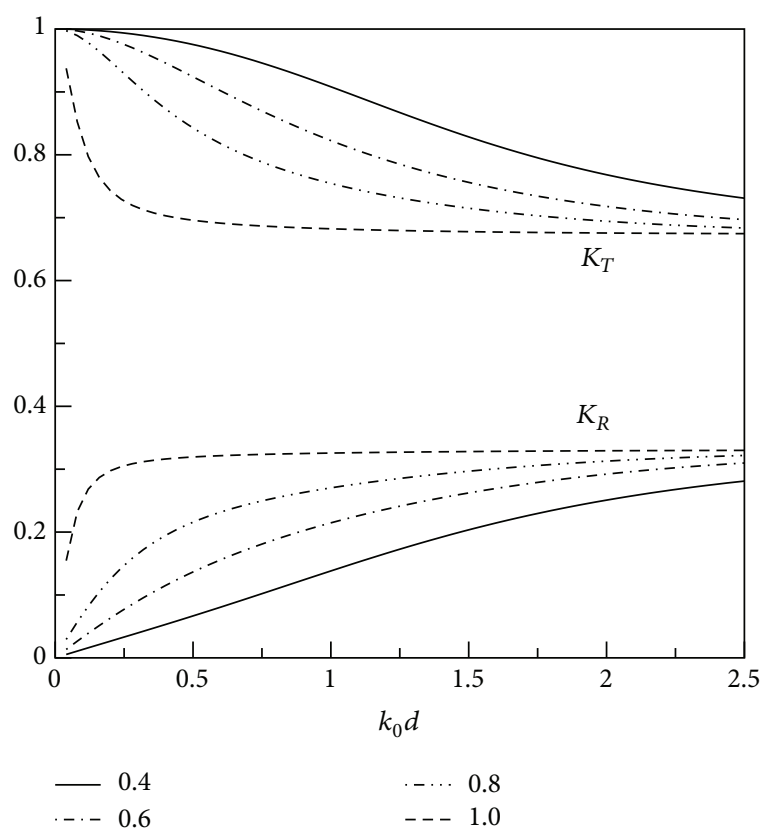

(a) The reflection and transmission coefficients, $K_{R}$ and $K_{T}$

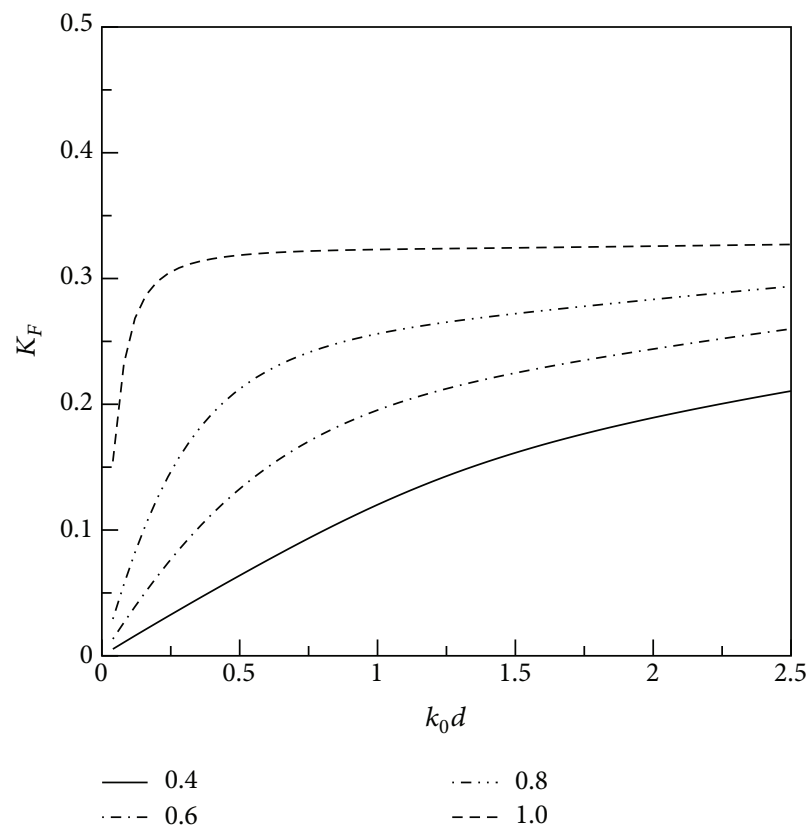

(b) The dimensionless wave force $K_{F}$

Figure 2: Variations of $K_{R}, K_{T}$, and $K_{F}$ versus $k_{0} d$ for a surfacepiercing porous barrier (Type 1 ) at $G=1+0.2 \mathrm{i}$ and different $a / d$.

accuracy at best. If we adopt larger $M$, the convergence is still slow. The reason is that, for the matched eigenfunction expansion solution, fluid velocity singularity near the edge of the porous barrier is not considered.

4.3. Theoretical Curves of Hydrodynamic Quantities. Figure 2 gives the variations of $K_{R}, K_{T}$, and $K_{F}$ for a surface-piercing porous barrier (Type 1 ) with dimensionless wave number $k_{0} d$ at a fixed porous effect parameter $G=1+0.2 \mathrm{i}$. It is observed 
TABLe 2: Results of $K_{R}, K_{T}$, and $K_{L}$ for Type 2 barrier at $G=0$.

\begin{tabular}{lccccc}
\hline$b / d$ & $k_{0} b$ & \multicolumn{2}{c}{ The present results } & \multicolumn{2}{c}{ Porter and Evans (1995) [1] } \\
& & $K_{R}$ & $K_{T}$ & $K_{L}$ & - \\
0.1 & 0.1 & 0.005598 & 0.999984 & 0 & $K_{R}$ \\
0.2 & 0.05 & 0.007817 & 0.999969 & 0 & 0.00782 \\
0.5 & 0.2 & 0.082591 & 0.996584 & 0 & - \\
0.6 & 0.3 & 0.150706 & 0.988579 & 0 & 0.15071 \\
0.9 & 1.8 & 0.542662 & 0.839951 & 0 & 0.94266 \\
\hline
\end{tabular}

TABLE 3: Results of the multi-term Galerkin solution for Type 1 porous barrier at $k_{0} a=0.6$ and $G=1+0.25 \mathrm{i}$.

\begin{tabular}{|c|c|c|c|c|c|c|c|c|c|}
\hline \multirow{2}{*}{$\begin{array}{l}\text { Truncated } \\
\text { number } N\end{array}$} & \multicolumn{3}{|c|}{$\frac{a}{d}=0.1$} & \multicolumn{3}{|c|}{$\frac{a}{d}=0.5$} & \multicolumn{3}{|c|}{$\frac{a}{d}=0.9$} \\
\hline & $K_{R}$ & $K_{T}$ & $K_{F}$ & $K_{R}$ & $K_{T}$ & $K_{F}$ & $K_{R}$ & $K_{T}$ & $K_{F}$ \\
\hline 0 & 0.222895 & 0.808722 & 0.139259 & 0.201558 & 0.835137 & 0.173011 & 0.267398 & 0.768831 & 0.261473 \\
\hline 1 & 0.223158 & 0.809090 & 0.139902 & 0.202015 & 0.835583 & 0.174000 & 0.271319 & 0.766307 & 0.266388 \\
\hline 2 & 0.223145 & 0.809113 & 0.139885 & 0.201999 & 0.835609 & 0.173977 & 0.271375 & 0.766303 & 0.266405 \\
\hline 3 & 0.223144 & 0.809114 & 0.139884 & 0.201998 & 0.835611 & 0.173976 & 0.271376 & 0.766305 & 0.266409 \\
\hline 4 & 0.223144 & 0.809114 & 0.139884 & 0.201998 & 0.835611 & 0.173976 & 0.271376 & 0.766305 & 0.266408 \\
\hline 5 & 0.223144 & 0.809114 & 0.139884 & 0.201998 & 0.835611 & 0.173976 & 0.271376 & 0.766306 & 0.266408 \\
\hline 6 & 0.223143 & 0.809114 & 0.139884 & 0.201998 & 0.835611 & 0.173975 & 0.271376 & 0.766306 & 0.266408 \\
\hline 7 & 0.223143 & 0.809114 & 0.139884 & 0.201998 & 0.835611 & 0.173975 & 0.271376 & 0.766306 & 0.266408 \\
\hline 9 & 0.223144 & 0.809114 & 0.139884 & 0.201998 & 0.835611 & 0.173975 & 0.271376 & 0.766306 & 0.266408 \\
\hline 10 & 0.223144 & 0.809114 & 0.139884 & 0.201998 & 0.835611 & 0.173975 & 0.271376 & 0.766306 & 0.266408 \\
\hline
\end{tabular}

TABLE 4: Results of the multi-term Galerkin solution for Type 2 porous barrier at $k_{0} b=0.2$ and $G=1+0.25 \mathrm{i}$.

\begin{tabular}{|c|c|c|c|c|c|c|c|c|c|}
\hline \multirow{2}{*}{$\begin{array}{l}\text { Truncated } \\
\text { number } N\end{array}$} & \multicolumn{3}{|c|}{$\frac{b}{d}=0.1$} & \multicolumn{3}{|c|}{$\frac{b}{d}=0.5$} & \multicolumn{3}{|c|}{$\frac{b}{d}=0.9$} \\
\hline & $K_{R}$ & $K_{T}$ & $K_{F}$ & $K_{R}$ & $K_{T}$ & $K_{F}$ & $K_{R}$ & $K_{T}$ & $K_{F}$ \\
\hline 0 & 0.003551 & 0.998938 & 0.007621 & 0.069920 & 0.975171 & 0.071480 & 0.175771 & 0.905392 & 0.176347 \\
\hline 1 & 0.003548 & 0.998943 & 0.007614 & 0.069879 & 0.975289 & 0.071435 & 0.177309 & 0.905034 & 0.177850 \\
\hline 2 & 0.003548 & 0.998943 & 0.007614 & 0.069878 & 0.975290 & 0.071434 & 0.177348 & 0.905026 & 0.177889 \\
\hline 3 & 0.003548 & 0.998943 & 0.007614 & 0.069878 & 0.975290 & 0.071434 & 0.177349 & 0.905026 & 0.177891 \\
\hline 4 & 0.003548 & 0.998943 & 0.007621 & 0.069878 & 0.975290 & 0.071434 & 0.177349 & 0.905026 & 0.177891 \\
\hline 5 & 0.003548 & 0.998943 & 0.007614 & 0.069878 & 0.975290 & 0.071434 & 0.177349 & 0.905026 & 0.177891 \\
\hline 6 & 0.003548 & 0.998943 & 0.007614 & 0.069878 & 0.975290 & 0.071434 & 0.177349 & 0.905026 & 0.177891 \\
\hline 7 & 0.003548 & 0.998943 & 0.007614 & 0.069878 & 0.975290 & 0.071434 & 0.177349 & 0.905026 & 0.177891 \\
\hline 9 & 0.003548 & 0.998943 & 0.007614 & 0.069878 & 0.975290 & 0.071434 & 0.177349 & 0.905026 & 0.177891 \\
\hline
\end{tabular}

TABLE 5: Results of the matched eigenfunction expansion solution for Type 1 porous barrier at $k_{0} a=0.6$ and $G=1+0.25$ i.

\begin{tabular}{|c|c|c|c|c|c|c|c|c|c|}
\hline \multirow{2}{*}{$\begin{array}{l}\text { Truncated } \\
\text { number } M\end{array}$} & \multicolumn{3}{|c|}{$\frac{a}{d}=0.1$} & \multicolumn{3}{|c|}{$\frac{a}{d}=0.5$} & \multicolumn{3}{|c|}{$\frac{a}{d}=0.9$} \\
\hline & $K_{R}$ & $K_{T}$ & $K_{F}$ & $K_{R}$ & $K_{T}$ & $K_{F}$ & $K_{R}$ & $K_{T}$ & $K_{F}$ \\
\hline 20 & 0.252275 & 0.771745 & 0.152222 & 0.209811 & 0.826342 & 0.180059 & 0.280401 & 0.754224 & 0.274120 \\
\hline 50 & 0.238389 & 0.789733 & 0.147991 & 0.205739 & 0.831183 & 0.177159 & 0.275419 & 0.760897 & 0.270155 \\
\hline 100 & 0.232329 & 0.797420 & 0.145332 & 0.204131 & 0.833086 & 0.175876 & 0.273561 & 0.763391 & 0.268507 \\
\hline 200 & 0.228312 & 0.802531 & 0.143226 & 0.203180 & 0.834212 & 0.175066 & 0.272607 & 0.764665 & 0.267320 \\
\hline 400 & 0.226035 & 0.805435 & 0.141870 & 0.202652 & 0.834837 & 0.174594 & 0.272048 & 0.765411 & 0.267082 \\
\hline 600 & 0.225193 & 0.806507 & 0.141332 & 0.202457 & 0.835067 & 0.174415 & 0.271847 & 0.765679 & 0.266885 \\
\hline
\end{tabular}


TABLE 6: Results of the matched eigenfunction expansion solution for Type 2 porous barrier at $k_{0} b=0.2$ and $G=1+0.25 \mathrm{i}$.

\begin{tabular}{|c|c|c|c|c|c|c|c|c|c|}
\hline \multirow{2}{*}{$\begin{array}{l}\text { Truncated } \\
\text { number } M\end{array}$} & \multicolumn{3}{|c|}{$\frac{b}{d}=0.1$} & \multicolumn{3}{|c|}{$\frac{b}{d}=0.5$} & \multicolumn{3}{|c|}{$\frac{b}{d}=0.9$} \\
\hline & $K_{R}$ & $K_{T}$ & $K_{F}$ & $K_{R}$ & $K_{T}$ & $K_{F}$ & $K_{R}$ & $K_{T}$ & $K_{F}$ \\
\hline 20 & 0.005142 & 0.998193 & 0.009922 & 0.076300 & 0.971694 & 0.077025 & 0.193357 & 0.889137 & 0.192532 \\
\hline 50 & 0.004270 & 0.998621 & 0.008808 & 0.072877 & 0.973634 & 0.074184 & 0.184363 & 0.898210 & 0.1814523 \\
\hline 100 & 0.003963 & 0.998760 & 0.008348 & 0.071573 & 0.974358 & 0.073031 & 0.181097 & 0.901417 & 0.181488 \\
\hline 200 & 0.003774 & 0.998844 & 0.008036 & 0.070812 & 0.974778 & 0.072333 & 0.179449 & 0.903013 & 0.179928 \\
\hline 400 & 0.003672 & 0.998889 & 0.007855 & 0.070393 & 0.975008 & 0.071937 & 0.178491 & 0.903934 & 0.179007 \\
\hline 600 & 0.003636 & 0.998905 & 0.007815 & 0.070239 & 0.975092 & 0.071790 & 0.178149 & 0.904262 & 0.178675 \\
\hline
\end{tabular}

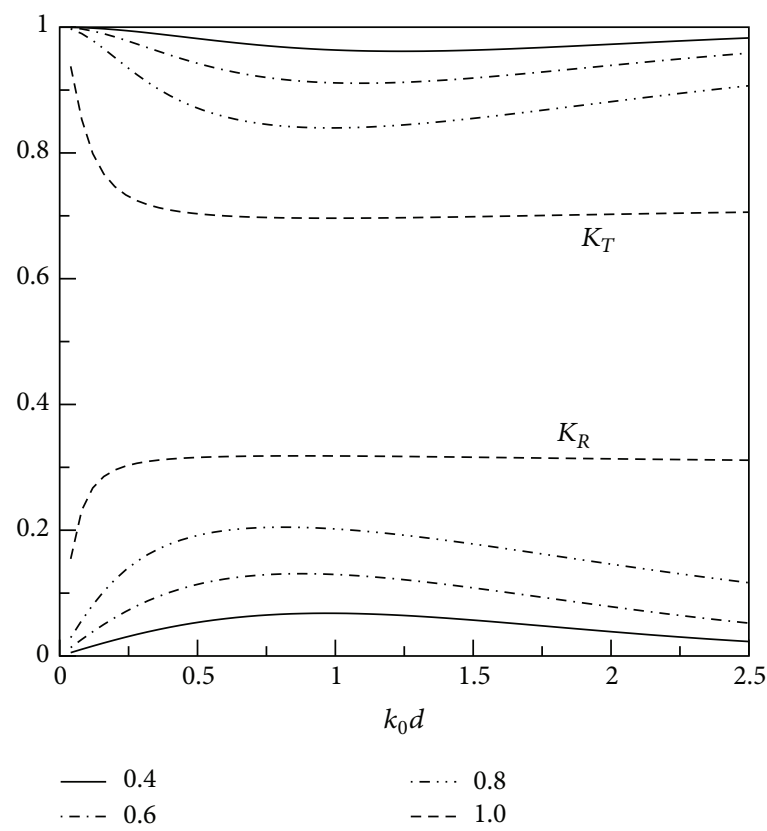

(a) The reflection and transmission coefficients, $K_{R}$ and $K_{T}$

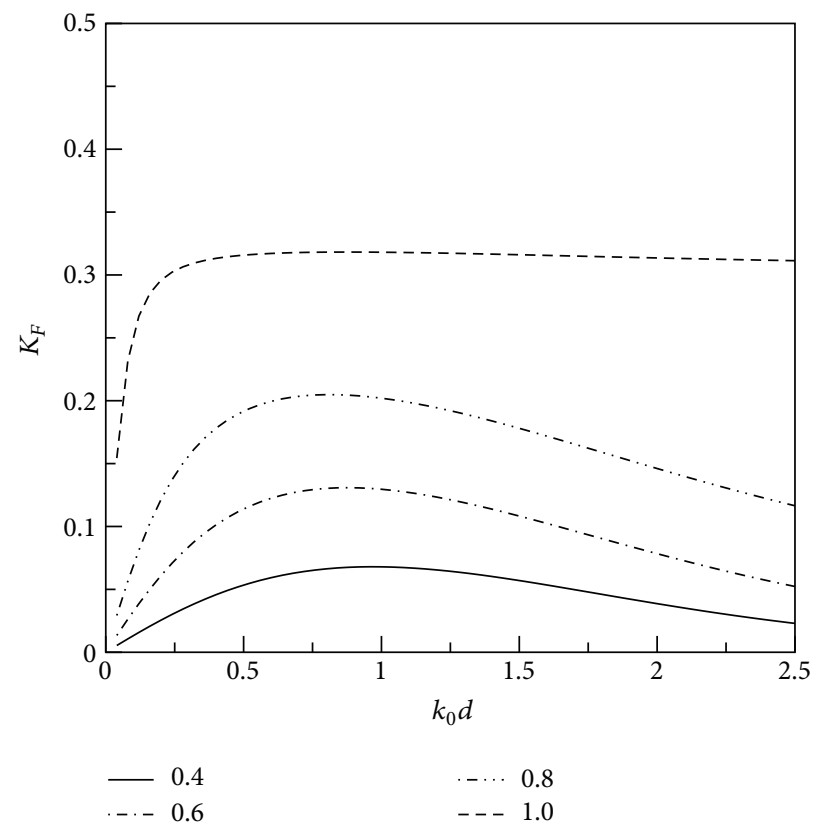

(b) The dimensionless wave force $K_{F}$

FIgURe 3: Variations of $K_{R}, K_{T}$, and $K_{F}$ versus $k_{0} d$ for a submerged porous barrier (Type 2) at $G=1+0.2 \mathrm{i}$ and different $b / d$.

that for a fixed relative barrier height $a / d, K_{R}$ and $K_{F}$ increase and $K_{T}$ decreases with the increasing value of $k_{0} d$. When the value of $k_{0} d$ is large enough, $K_{R}, K_{T}$, and $K_{F}$ all tend constants. It can also be observed from Figure 2 that, for a fixed $k_{0} d$, the reflection coefficient and the wave force both decease with the decreasing relative barrier height $a / d$, while the transmission coefficient increases with the deceasing $a / d$.

Figure 3 shows the variations of $K_{R}, K_{T}$, and $K_{F}$ for a submerged porous barrier (Type 2) with dimensionless wave number $k_{0} d$ at a fixed porous effect parameter $G=1+0.2 \mathrm{i}$. It can be seen that the variations of the dimensionless wave force $K_{F}$ of Type 2 barrier with the relative barrier height $b / d$ are similar to those of Type 1 barrier. However, the reflection and transmission coefficients attain, respectively, a maximum value and minimum value with the increasing wave number $k_{0} d$. This is in fact a common feature for wave motion over submerged barriers.

Finally, we examine the effects of the porous effect parameter $G$ on $K_{R}, K_{T}$, and $K_{F}$. For Type 1 porous barrier, we fix the relative barrier height at $a / d=0.5$ and illustrate the results of $K_{R}, K_{T}$ and $K_{F}$ at different values of $G$ in Figure 4 . When the porous effect parameter $G$ increases at a fixed $k_{0} d$, the reflection coefficient and the wave force on the barrier both decrease significantly. When $G=1$ and $k_{0} d<1.2$, the transmission and reflection coefficients of porous barrier are both smaller than those of impermeable barrier. This is due to the additional wave energy dissipation caused by the holes in porous barrier. For Type 2 porous barrier, we fix the relative barrier height at $b / d=0.5$ and give the results of $K_{R}, K_{T}$, and $K_{F}$ at different values of $G$ in Figure 5. It can be seen that the effects of $G$ on the $K_{R}$ 


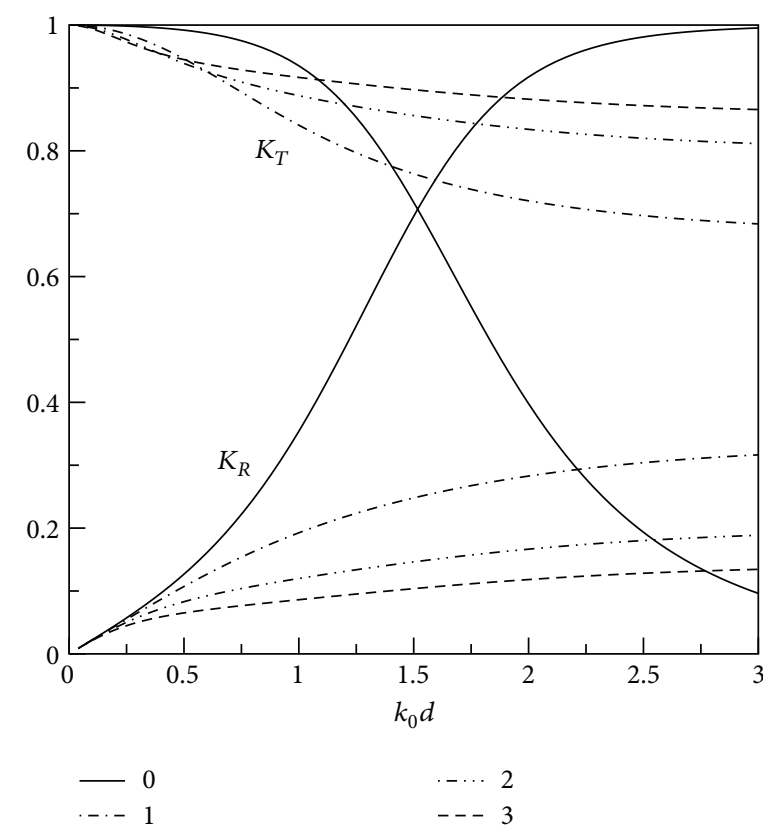

(a) The reflection and transmission coefficients, $K_{R}$ and $K_{T}$

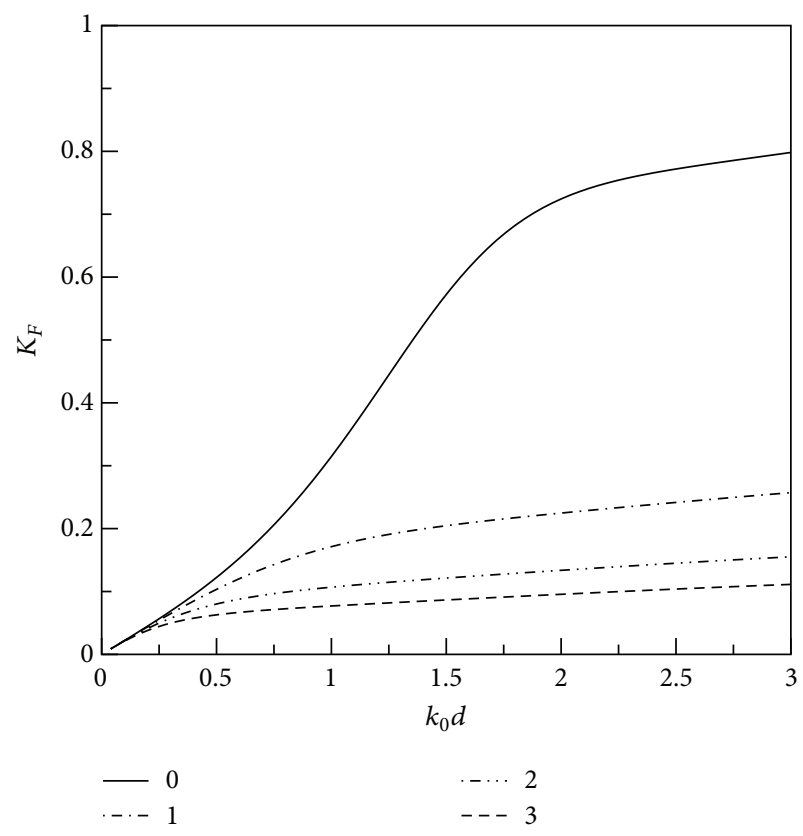

(b) The dimensionless wave force $K_{F}$

FIGURE 4: Variations of $K_{R}, K_{T}$, and $K_{F}$ versus $k_{0} d$ for a surface-piercing porous barrier (Type 1 ) at $a / d=0.5$ and different $G$.

and $K_{F}$ of Type 2 barrier are similar to those of Type 1 barrier.

\section{Conclusions}

This study has developed accurate solutions for water wave scattering by two types of thin vertical porous barriers based on the linear potential theory. The multi-term Galerkin method has been used to take into account the square-root singularity near the edge of the porous barrier. We have shown that the multi-term Galerkin method worked very well for porous barriers. The convergence of the present solution was very rapid in comparison with the traditional matched eigenfunction expansion solution. Accurate results of various hydrodynamic quantities have been presented. The reflection and transmission coefficients of porous barrier may be both smaller than those of impermeable barrier. The wave force acting on a porous barrier is significantly reduced compared to the corresponding solid barrier. This should be favorable in some coastal engineering applications.
The present study gives a reliable benchmark for other numerical solutions with respect to porous thin walls. Most of all, this study provides a promising procedure for solving wave interaction with vertical thin porous barriers.

\section{Appendix}

\section{Matched Eigenfunction Expansion Solution}

According to (17) and (18), we define a new function:

$$
\begin{aligned}
& H(z) \\
& \quad= \begin{cases}\left.\left(\phi_{1}-\phi_{2}\right)\right|_{x=0}=0, & z \in \Lambda, \\
{\left.\left[\frac{\partial \phi_{2}}{\partial x}-\mathrm{i} k_{0} G\left(\phi_{1}-\phi_{2}\right)\right]\right|_{x=0}=0,} & z \in \Gamma .\end{cases}
\end{aligned}
$$

Substituting the expressions for the velocity potentials in (11) with (20) into (A.1), we have

$$
H(z)= \begin{cases}2 R Z_{0}(z)+2 \sum_{m=1}^{\infty} A_{m} Z_{m}(z)=0, & z \in \Lambda, \\ \mathrm{i} k_{0}[1-(2 G+1) R] Z_{0}(z)+\sum_{m=1}^{\infty}\left(k_{m}-2 \mathrm{i} k_{0} G\right) A_{m} Z_{m}(z)=0, & z \in \Gamma .\end{cases}
$$

Multiplying both sides of (A.2) by $Z_{n}(z)$, integrating with respect to $z$ over $[-d, 0]$, and then truncating $n$ and $m$ after $M$ terms, we have

$$
\begin{aligned}
& \left\{\left[a_{n m}\right]_{(M+1) \times(M+1)}+\left[b_{n m}\right]_{(M+1) \times(M+1)}\right\}\left\{A_{m}\right\}_{(M+1)} \\
& \quad=\left\{f_{n}\right\}_{(M+1)},
\end{aligned}
$$




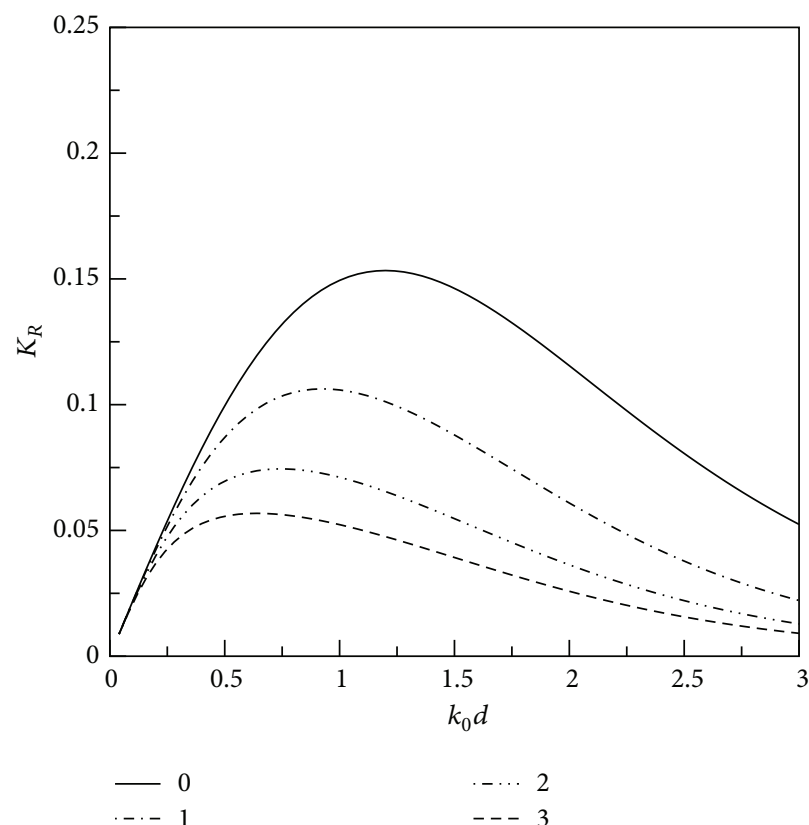

(a) The reflection coefficient $K_{R}$

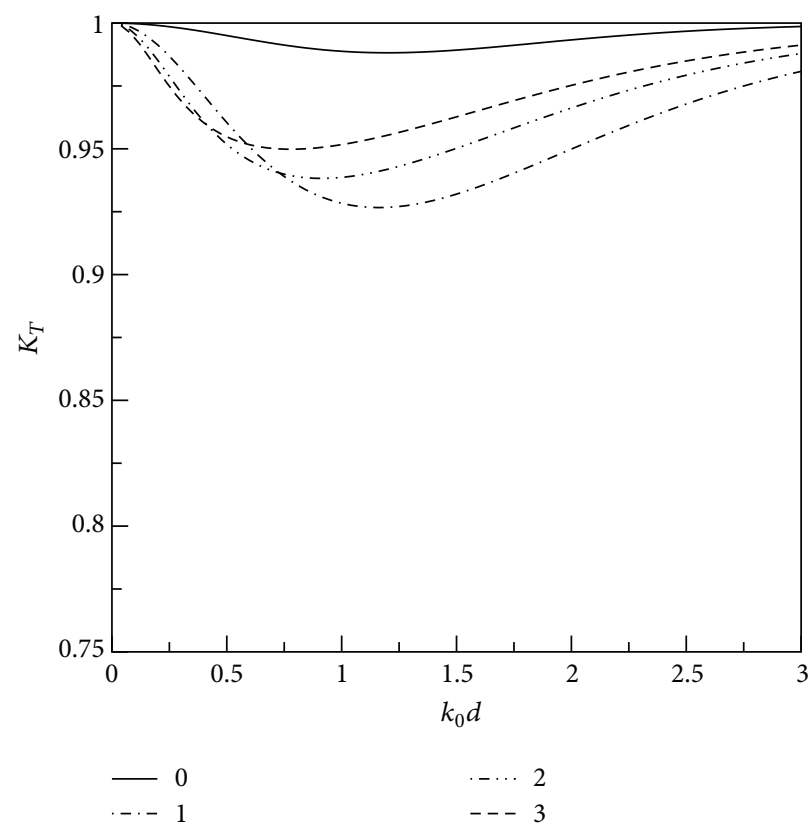

(b) The transmission coefficient $K_{T}$

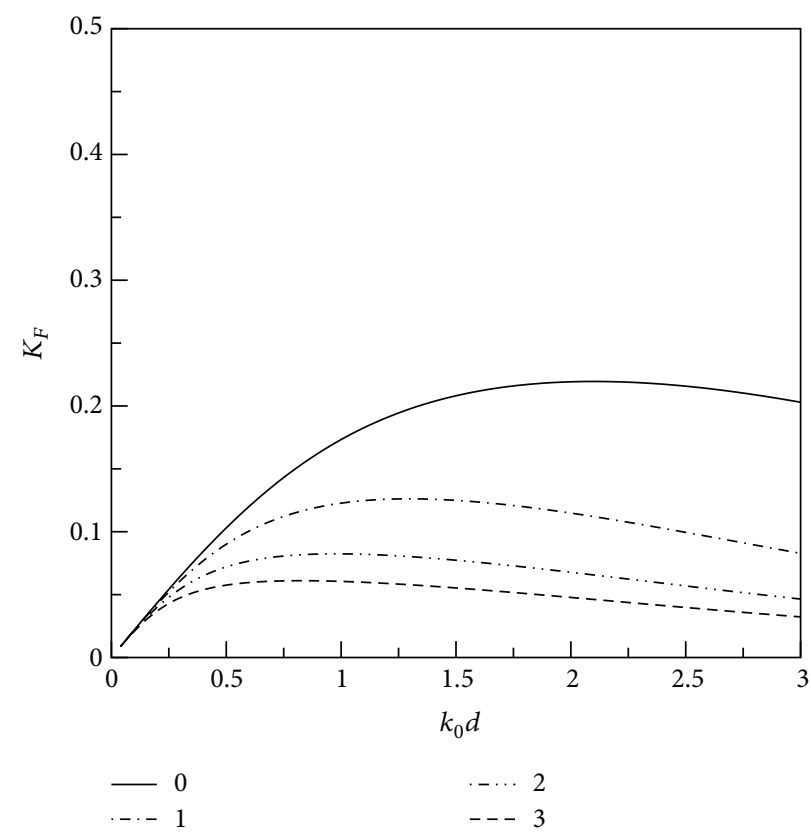

(c) The dimensionless wave force $K_{F}$

FIgURE 5: Variations of $K_{R}, K_{T}$, and $K_{F}$ versus $k_{0} d$ for a submerged porous barrier (Type 2) at $b / d=0.5$ and different $G$.

where

$$
\begin{aligned}
f_{n} & =\mathrm{i} k_{0} \int_{\Gamma} Z_{0}(z) Z_{n}(z) \mathrm{d} z, \quad n=0,1, \ldots, M, \\
a_{n m} & =2 \int_{\Lambda} Z_{m}(z) Z_{n}(z) \mathrm{d} z, \quad m, n=0,1, \ldots, M, \\
b_{n 0} & =\mathrm{i} k_{0}(1+2 G) f_{n}, \quad n=0,1, \ldots, M,
\end{aligned}
$$

$$
b_{n m}=\left(k_{m}-2 \mathrm{i} k_{0} G\right) \int_{\Gamma} Z_{m}(z) Z_{n}(z) \mathrm{d} z
$$$$
m=1,2, \ldots, M, n=0,1, \ldots, M \text {, }
$$

$$
A_{0}=R
$$

The unknown coefficients $A_{m}$ are obtained after solving (A.3). Then, the velocity potentials and various hydrodynamic quantities are determined. 


\section{Conflict of Interests}

The authors declare no conflict of interests.

\section{Acknowledgment}

This work was supported by the National Natural Science Foundation of China (Grant nos. 51322903 and 51279224).

\section{References}

[1] R. Porter and D. V. Evans, "Complementary approximations to wave scattering by vertical barriers," Journal of Fluid Mechanics, vol. 294, pp. 155-180, 1995.

[2] M. Isaacson, S. Premasiri, and G. Yang, "Wave interactions with vertical slotted barrier," Journal of Waterway, Port, Coastal and Ocean Engineering, vol. 124, no. 3, pp. 118-126, 1998.

[3] K.-D. Suh, S. Shin, and D. T. Cox, "Hydrodynamic characteristics of pile-supported vertical wall breakwaters," Journal of Waterway, Port, Coastal and Ocean Engineering, vol. 132, no. 2, pp. 83-96, 2006.

[4] H. Oumeraci and G. Koether, "Hydraulic performance of a submerged wave absorber for coastal protection," in Nonlinear Wave Dynamics, P. Lynett, Ed., pp. 31-65, World Scientific Publishing, Hackensack, NJ, USA, 2009.

[5] Z. H. Huang, Y. C. Li, and Y. Liu, "Hydraulic performance and wave loadings of perforated/slotted coastal structures: a review," Ocean Engineering, vol. 38, no. 10, pp. 1031-1053, 2011.

[6] F. Ursell, "The effect of a fixed vertical barrier on surface waves in deep water," Proceedings of the Cambridge Philosophical Society, vol. 43, no. 3, pp. 374-382, 1947.

[7] D. V. Evans, "Diffraction of water waves by submerged vertical plate," Journal of Fluid Mechanics, vol. 40, no. 3, pp. 433-451, 1970.

[8] C. A. N. Morris, "A variational approach to an unsymmetric water-wave scattering problem," Journal of Engineering Mathematics, vol. 9, no. 4, pp. 291-300, 1975.

[9] I. J. Losada, M. A. Losada, and A. J. Roldán, "Propagation of oblique incident waves past rigid vertical thin barriers," Applied Ocean Research, vol. 14, no. 3, pp. 191-199, 1992.

[10] A. G. Abul-Azm, "Wave diffraction through submerged breakwaters," Journal of Waterway, Port, Coastal and Ocean Engineering, vol. 119, no. 6, pp. 587-605, 1993.

[11] D. L. Kriebel and C. A. Bollmann, "Wave transmission past vertical wave barriers," in Proceeding of the 25th Coastal Engineering Conference, pp. 2470-2480, ASCE, Orlando, Fla, USA, September 1996.

[12] S. Banerjea, M. Kanoria, D. P. Dolai, and B. N. Mandal, "Oblique wave scattering by submerged thin wall with gap in finite-depth water," Applied Ocean Research, vol. 18, no. 6, pp. 319-327, 1996.

[13] D. V. Evans and R. Porter, "Efficient calculation of hydrodynamic properties of OWC-type devices," Journal of Offshore Mechanics and Arctic Engineering, vol. 119, no. 4, pp. 210-218, 1997.

[14] H. Martins-Rivas and C. C. Mei, "Wave power extraction from an oscillating water column at the tip of a breakwater," Journal of Fluid Mechanics, vol. 626, pp. 395-414, 2009.

[15] K.-H. Chang, D.-H. Tsaur, and L.-H. Huang, "Accurate solution to diffraction around a modified V-shaped breakwater," Coastal Engineering, vol. 68, pp. 56-66, 2012.
[16] E. O. Tuck, "Matching problems involving flow through small holes," Advances in Applied Mechanics, vol. 15, pp. 89-158, 1975.

[17] C. Macaskill, "Reflexion of water waves by a permeable barrier," Journal of Fluid Mechanics, vol. 95, no. 1, pp. 141-157, 1979.

[18] T. Sahoo, "On the scattering of water waves by porous barriers," Zeitschrift für Angewandte Mathematik und Mechanik, vol. 78, no. 5, pp. 364-370, 1998.

[19] M. M. Lee and A. T. Chwang, "Scattering and radiation of water waves by permeable barriers," Physics of Fluids, vol. 12, no. 1, pp. 54-65, 2000.

[20] T. Sahoo, A. T. Chan, and A. T. Chwang, "Scattering of oblique surface waves by permeable barriers," Journal of Waterway, Port, Coastal and Ocean Engineering, vol. 126, no. 4, pp. 196-205, 2000.

[21] D. Karmakar and C. Guedes Soares, "Wave transformation due to multiple bottom-standing porous barriers," Ocean Engineering, vol. 80, pp. 50-63, 2014.

[22] R. Gayen and A. Mondal, "A hypersingular integral equation approach to the porous plate problem," Applied Ocean Research, vol. 46, pp. 70-78, 2014.

[23] C. M. Linton and P. McIver, Handbook of Mathematical Techniques for Wave/Structure Interactions, Chapman \& Hall/CRC, Boca Raton, Fla, USA, 2001.

[24] D. V. Evans and M. A. Peter, "Asymptotic reflection of linear water waves by submerged horizontal porous plates," Journal of Engineering Mathematics, vol. 69, no. 2-3, pp. 135-154, 2011.

[25] X. P. Yu, "Diffraction of water waves by porous breakwaters," Journal of Waterway, Port, Coastal and Ocean Engineering, vol. 121, no. 6, pp. 275-282, 1995.

[26] C. K. Sollitt and R. H. Cross, "Wave transmission through permeable breakwaters," in Proceedings of the 13th Coastal Engineering Conference, pp. 1827-1846, Vancouver, BC, Canada, 1972.

[27] Y. C. Li, Y. Liu, and B. Teng, "Porous effect parameter of thin permeable plates," Coastal Engineering Journal, vol. 48, no. 4, pp. 309-336, 2006.

[28] K.-D. Suh, Y. W. Kim, and C.-H. Ji, "An empirical formula for friction coefficient of a perforated wall with vertical slits," Coastal Engineering, vol. 58, no. 1, pp. 85-93, 2011.

[29] I. N. Sneddon, Mixed Boundary Value Problems in Potential Theory, North Holland, Amsterdam, The Netherlands, 1966.

[30] R. A. Dalrymple and P. A. Martin, "Wave diffraction through offshore breakwaters," Journal of Waterway, Port, Coastal and Ocean Engineering, vol. 116, no. 6, pp. 727-741, 1990.

[31] A. J. Roldan, M. A. Losada, and I. J. Losada, "Theoretical study of the wave attenuation in a channel with roughened sides," in Proceedings of the 23rd Coastal Engineering Conference, pp. 2786-2798, Venice, Italy, 1992.

[32] Y. Liu and Y.-C. Li, "Wave interaction with a wave absorbing double curtain-wall breakwater," Ocean Engineering, vol. 38, no. 10, pp. 1237-1245, 2011. 


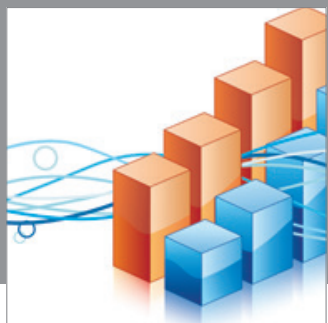

Advances in

Operations Research

mansans

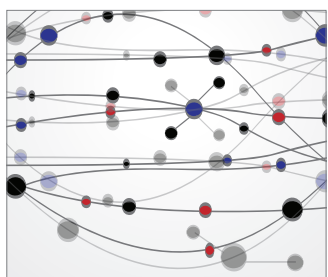

The Scientific World Journal
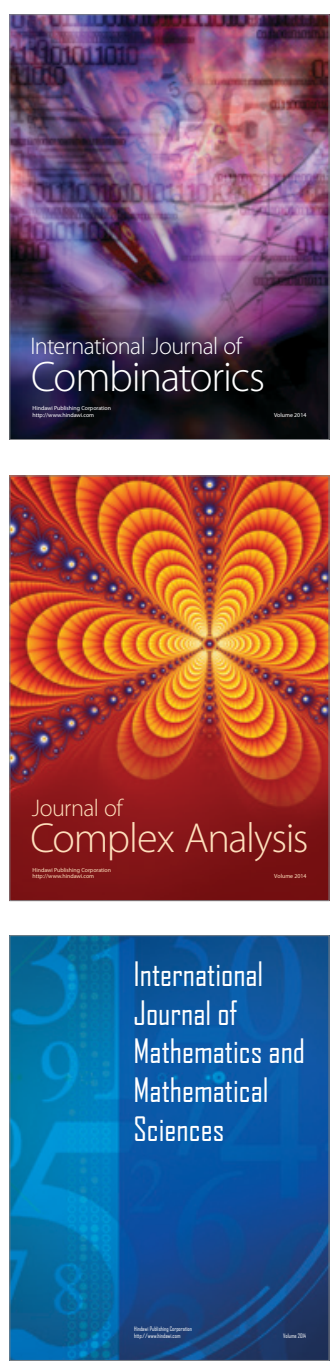
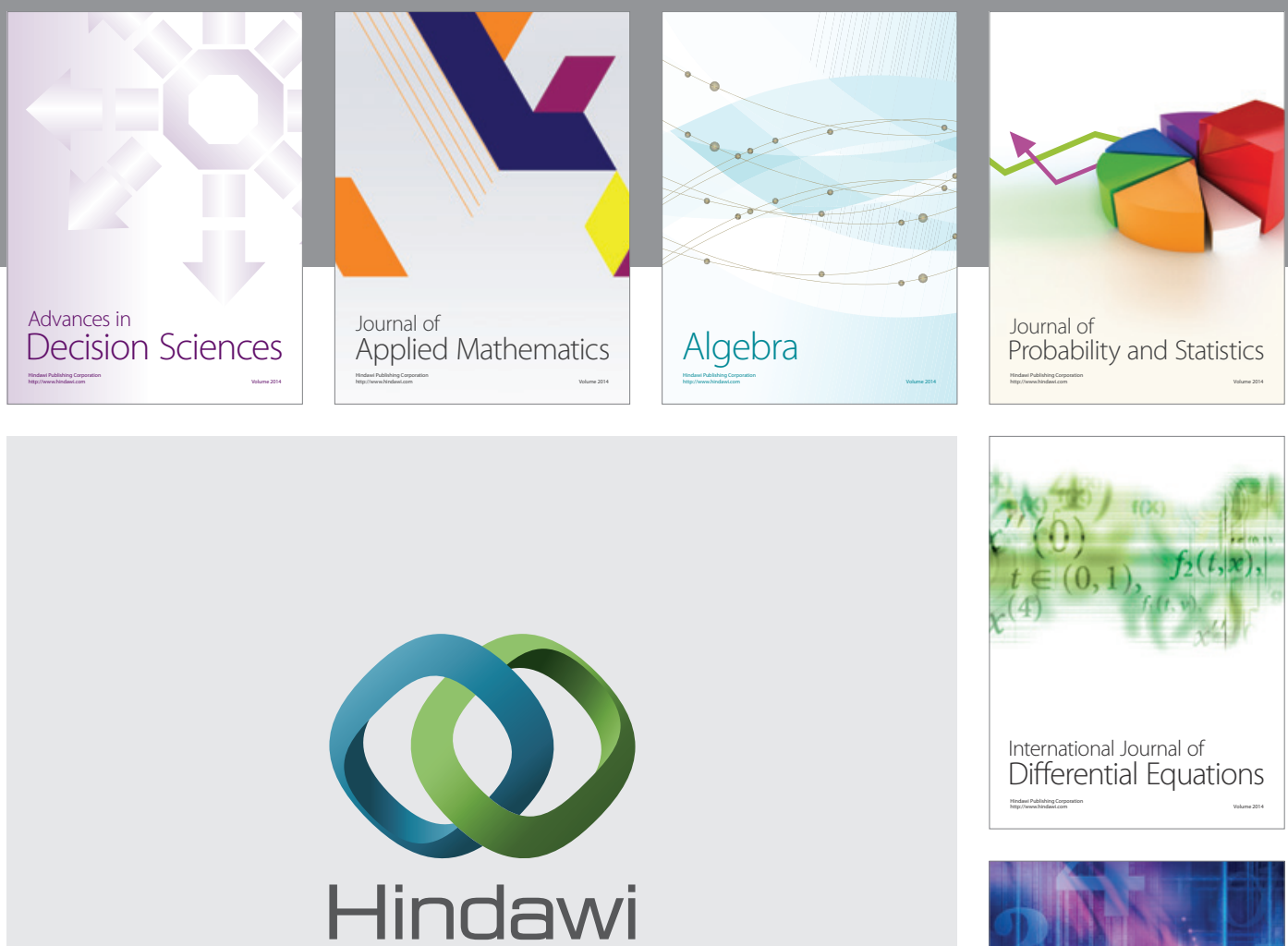

Submit your manuscripts at http://www.hindawi.com
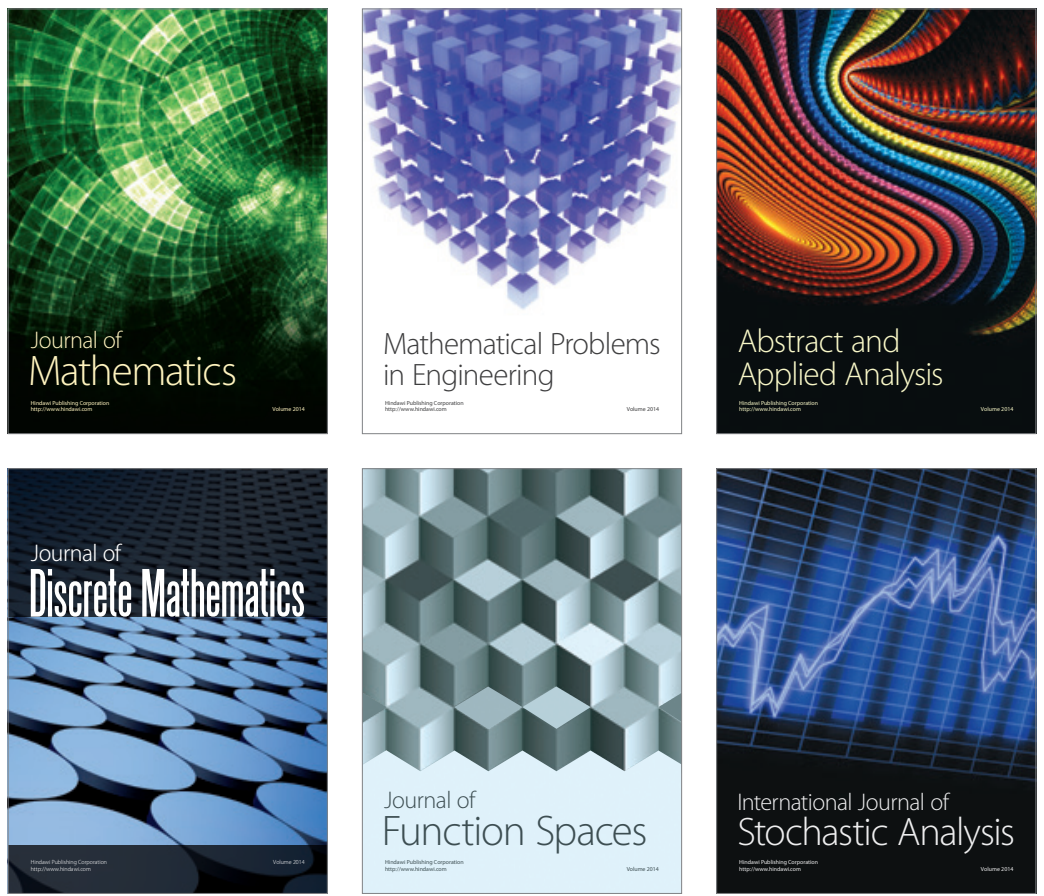

Journal of

Function Spaces

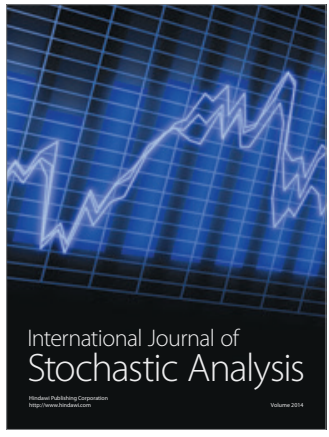

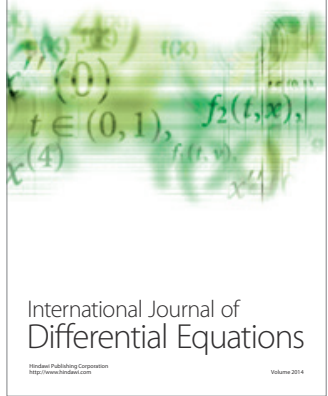
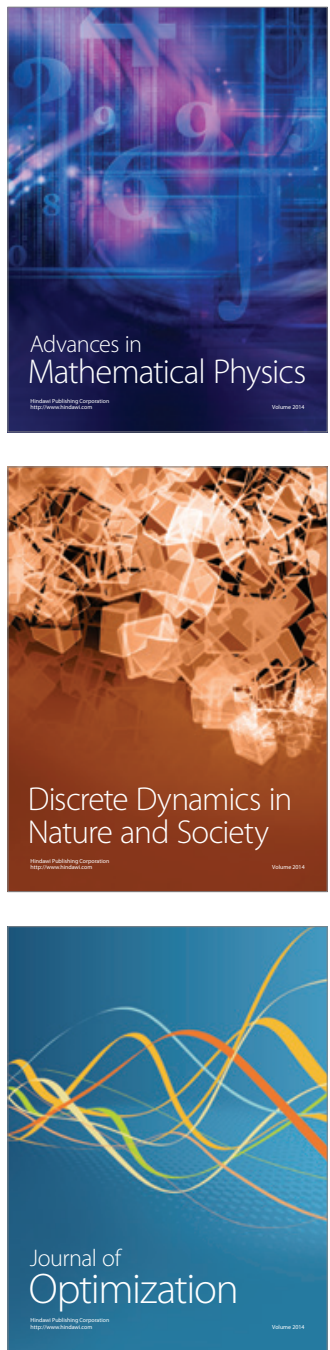